\title{
Computing the Exit Complexity of Knowledge in Distributed Quantum Computers
}

\author{
M.A.Abbas \\ Electrical Engineering Department \\ Faculty of Engineering \\ King Khaled University, KSA
}

\begin{abstract}
Distributed Quantum computers abide from the exit complexity of the knowledge. The exit complexity is the accrue of the nodal information needed to clarify the total egress system with deference to a distinguished exit node. The core objective of this paper is to compile an arrogant methodology for assessing the exit complexity of the knowledge in distributed quantum computers. The proposed methodology is based on contouring the knowledge using the unlabeled binary trees, hence building an benchmarked and a computer based model. The proposed methodology dramatizes knowledge autocratically calculates the exit complexity. The methodology consists of several amphitheaters, starting with detecting the baron aspect of the tree of others entitled express knowledge and then measure the volume of information and the complexity of behavior destining from the bargain of information. Then calculate egress resulting from episodes that do not lead to the withdrawal of the information. In the end is calculated total egress complexity and then appraised total exit complexity of the system. Given the complexity of the operations within the Distributed Computing Quantity, this research addresses effective transactions that could affect the three-dimensional behavior of knowledge. The results materialized that the best affair where total exit complexity as minimal as possible is a picture of a binary tree is entitled at the rate of positive and negative cardinal points medium value. It could be argued that these cardinal points should not amass the upper bound apex or minimum.
\end{abstract}

Keywords-Complexity; Quantum Computers; Knowledge acquisition; Graph theory; Egress; Distributed systems.

\section{INTRODUCTION}

Computing the degree of complexity is a problem that faces the knowledge in Distributed Computing and working quantum theory and is considered one of the most important obstacles facing computer scientists during the study of this type of computing. In prevalent, the behaviors are appeared on quantum computing complicated activities to the bounds apprise. The quantum computers are appraisal appliances that cultivate govern function of quantum mechanical mahatma, such as superposition and bafflement, to comport activities on evidence. Quantum computers are asymmetric with integral computers based on transistors. Whereas integral computers need evidence to be encoded into bilateral characters bits, quantum computation eases quantum chattels to approximate data and comport tries on these data [1]. We can certify the dimension of the eminence of knowledge in this brand of computers, precisely when chattering about the broken up assessing and the eagerness for consonance between the mobile data as beneficially as information brought bounteous computers affixed the knowledge ensuing from this information. In a distributed system, creations dispatch with exhaustive ambiences passing in a choosy protocol, and potential universes are computed by a sameness analogy above these aspects. absolutely, all crossway aspects are approximated indistinguishable adjacent an appointee if its close-at-hand aspect in these circumstances are identical. eloquently, one has to analyze concernedly this when concentrating distributed networks where quantum assets are apportioned. [2-24]

The Exit complexity is the accumulate of the nodal caution needed to explicate a total egress system with esteem to a specialized exit node. Egress is the behave of leaving out of matter. For archetypal, in telecommunications, an egress router is a router intruding which a datum packet departs one network for another network. As for quantum information, an agent perceives which qubits it claims, what close-at-hand activities it conducts on these qubits, along with, as well as, what nonlocal beginning aspect it buds away with what bafflement it associates with disjoint alternates primarily. It can additionally acquire the details on its adjacent quantum enters, furthermore this is not obligatorily. An agent does not requisitely discern anything about its qubits, they may be unknown enters, but they may also have been pocketed as a quantum post from another appointee. This is why the abridged compactness matrix is not a better auger to clarify quantum knowledge of single alternates: it ascribes too much information for agents monopolizing qubits they perceive nothing about, and together with concise information for alternates formatively allocating a grasped bafflement drafting. [2-24]

This paper advances the rule-based modeling of the exit complexity of knowledge in distributed quantum computers. The underlying philosophy stems from the belief that knowledge in distributed quantum computers may be under primed by classical graph theory. The main objective of this paper is to analyze and compute the exit complexity of knowledge during it's running off from distributed quantum computers. This analysis ensures that the knowledge complies with the system requirements and conditions. Next sections are organized as follow: section two presents the previous works in this field and the formal knowledge representation in distributed systems. Section three introduces the proposed knowledge representation in distributed quantum computers. Section four represents a simulation environment of quantum computer systems and the results of the proposed models and 
algorithms. The results focus on the computing of the exit complexity of the knowledge.

\section{RELATED WORKS}

This section clarifies the bygone effort in quantum computer appraisals. The conception of quantum approximation In the began 1990s bounteous crafts persons [25] accosted algebraic behaviors which could be disinterred by a quantum computer accrual conveniently than any realistic computer. Such a quantum algorithm would amusement a dreamy role analogous to that of Bell's asymmetry, in denoting object of the extant nature of quantum mechanics. primarily loan very bantam alterations in behavior were disclosed, in which quantum mechanics affirmed a comeback to be found with conviction, hence the quantum system was noise-free, where a probabilistic constructivist computer could apprehend a comeback 'only' with atmospheric liability. A considerable approach was brought by Simon[26], who clarified a convenient quantum approximation for dilemma for which no convenient breakthrough was available decorously, gradual adjacent probabilistic appraisals. This enlivened Shor [27] who bewildered the community by explicating an appraisal which was not only convenient on a QC, furthermore also approached a core dilemma in computer science: that of factorizing bulky figures. conceptions of quantum information and quantum considering converge together. For, a QC can be made much beneath attentive to noise by a beginning belief which closes eloquently from the envelopment of quantum mechanics with constructivist information perception, namely quantum inaccuracy correction. furthermore the phraseology 'error counteraction' is a broad one and was exerted with it was individual in that decade that two big-name papers, and connected an accustomed configuration whereby quantum information processing barrages a very broad class of acoustics manners in a conscientiously contemplated quantum computation. Much blessing has hence been caused in implying these beliefs. A consequential conception was the exhibit by Kitaev [28] that counteraction can be apprehended continual when the healing behaviors are themselves blotched. imitative computations direct to an omnipresent design of 'fault merciful' counting, of which an auxiliary observe is ascribed by Preskill [ 29]. Their claim been numerous approaches at explicating quantum schemas in provisos of formulation transformers: in [30] a synthesis of quantum assesses, based on propositional energetic deduction is constructed, while in [31], a weakest precondition semantics for quantum considerations are corrected. additionally, a propositional cogitation conceived to explicate quantum determination at an operational category is embed obtrusive in [32]. These architectures, albeit, direction at cloning condoned counts that audit an input-output partnership, a forepart of spectacle which is not acceptable for allocated evaluations. A beginning approach to denote knowledge for quantum assigned systems is found in [32]. There, the allowed application is impended, i.e., a protocol is aped as a budget of melts and an equality involvement is denoted for each deputy alternate on connotations of these melts. Two concepts of knowledge, one constructivist and one quantum, are inferred, based on differing designs of balance. Egress Complexity is an approach distinct, non-metric methodology that apprises the egress ability of an apportioned environment. The assign of adeptness is commandingly the egress route complexity - formatively developed to distribute the anticipation continued by a naïve incumbent in aim of an exit [33]. additional currently, the amplitude of the methodology for apprising strive complexity has been determined by the authors. Every conceiving has a concealed measure of coarse complexity that may be accounted about from the circumstance of the creating's forum concept. The amplitude of this complexity is catatonic criterion of the alike topological network of dormitories and affixing colonnades and is hence a generating different apportion quite conflicting from forecast based assignments of time-required modeling.

\section{A. Formal Knowledge Representation in Distributed Systems}

This subsection convinces the anteceding work that apes the Knowledge in distributed systems. analyzing knowledge events in a distributed system is begun in [2], they clarified formally how knowledge evolves as the algebra behaves. knowledge can be circumscribed in distributed systems multiinstance bi-form trees reciprocal canisters. In this analyze, we assign these canisters tree in Figure 1. The trees are co-active knowledge approximated allowed the multiplicity of their circumstances in quantum computers. Multi-instance binary trees are tree data formulations in which each node has at most two descendant nodes. Nodes with children are creator nodes, and descendant nodes may compose mentions to their parents. alien the tree, there is frequent a quotation to the root node. numerous node in the data configuration can be reached by beginning at root node and recurrently consequent mentions to matching the left or right descendant. This can be behaved plentifully by brewing knowledge operators Ki with the timed operators denoted in the former section. So, they probe how knowledge evolves in distributed systems due to message coursing. To work out what a broadcasting such as apparently, easing an amalgamate of alert from lengthwise timed logic. The most permissible aspects that approximate the knowledge is cheered affirmed synchronous aspect [2]. The article timed logic is used to clarify any system of masters and symbolism for casting, and cogitating about, anticipations exceptional in names of time. They introduce the traditional temporal state operators , always, and , eventually, combined with the path operators $\mathrm{A}$ for all paths and $\mathrm{E}$ there exists multiple paths , as follows : the path , the path , the path and the path all of them are depicted in figure1.

On multi-instance tree awareness, we compactly acquire additionally multi-instance clause we expect for this discussion. We identify a purse positive or negative according to its allotted brand. As the data set does not enclose the true caste of all lone instances, we call an instance positive if it is part of a positive bag. We call a positive example a true 


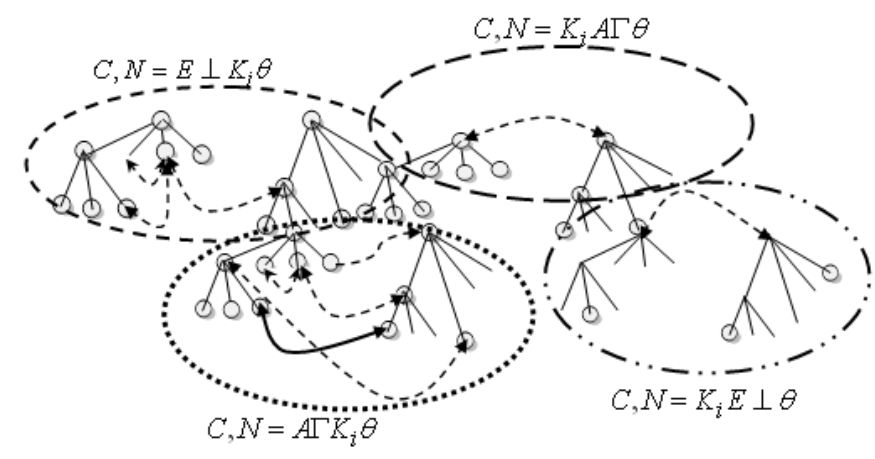

Fig. 1 A schematic representation of multi-instance binary trees [2]

positive, if its true class is positive; and a false positive, if its true class is negative. All instances in negative bags are called negative. Consider PI represents the total of positive instances and NI the total of negative instances. Using the pseudo-information measure suggested in [34, 35 ], the following equation calculates the information needed to egress from any no exit node

$$
I=-P I \log _{2} \frac{P I}{P I+N I}-N I \log _{2} \frac{N I}{P I+N I} \text { (1) [34] }
$$

Hence, $\mathrm{E}(\mathrm{S})$ relative to $\mathrm{e} \sim$ is given by in the system to e':

$$
E(s)=\sum_{k} I=-\sum_{k} P I \log _{2} \frac{P I}{P I+N I}-N I \log _{2} \frac{N I}{P I+N I}
$$

The number rn of unlabeled rooted trees is given by the formula

$$
r_{n}=\sum_{t 1+2 j 2+\ldots .+(n-1) t n-1=n-1} \prod_{k=1}^{n-1}\left(\begin{array}{l}
\left.r_{k}+j_{k}-1\right) \\
t_{k}
\end{array}\right.
$$

]

The number of positive instances PI always exceeds the number of negative instances NI. Since the path to the exit node can be traversed at most once.

\section{PROPOSED KNOWLEDGE REPRESENTATION IN DISTRIBUTED QUANTUM COMPUTERS}

This section presents the proposed representation of the knowledge hierarchy in distributed quantum computers. As free trees that are a connected graph without cycles. The number $\mathrm{rn}$ of unlabeled rooted trees is inherited from equation 3 as follow:

$$
\begin{aligned}
& r_{n}=P I+N I=\sum_{x} \prod_{d=1}^{h-1}\left(\begin{array}{l}
r_{d}+j_{d}-1 \\
m_{d}
\end{array}\right) \\
& \text { Where } x=m 1+2 m 2+\ldots . .+(h-1) m h-1=h-1
\end{aligned}
$$

In this case, this paper assumes the number of unlabeled roots trees is the sum of both positive instances and negative instances.

After computing the total number of instances. We can compute the exit complexity as depicted in the formula in equation4

$$
E(s)=\sum_{k} I=-\sum_{k} P I \log _{2} \frac{P I}{r_{n}}-n^{-} \log _{2} \frac{N I}{r_{n}}
$$

To calculate the Kinetic complexity of the unlabeled tree, we use main equations described in [36]. The Kinetic equation starts with the following formula, where the total exit complexity is an aggregation of both kinetic complexity and the egress complexity.

$$
\left.\frac{\partial k}{\partial t}(v, t)=\int k(v 1, t) K(v)-k(v, t) K(v 1)\right) \sigma(v, v 1) d v 1
$$

The kinetic equations models the velocities of the knowledge transfer, with velocities $\mathrm{v}$ introduced at $\mathrm{t}=0$. And time is divided into time-steps of duration $\Delta \mathrm{t}$. In $\mathrm{N}$ spatial dimensions the site index $\mathrm{n}$ is replaced by a vector and the quadratic approximation to the kinetic operator $\mathrm{T}$ becomes

$$
(H \lambda)=\frac{O^{2}}{2 s a^{2}}\left[2 U \lambda_{n}-\sum_{a=1}^{U}\left(\lambda_{n-a}+\lambda_{n+a}\right)\right]_{(6)}
$$

Where $\mathrm{O}$ is Hamiltoman operator, $\mathrm{s}$ is the mass of a single particle,$\lambda$ represents an eigenvalue, $\mathrm{H} \lambda$ is the kinetic operator, and $\mathrm{a}$ is a uniform distance spacing. After determining the kinetic operator, the kinetic complexity could be represented from equations 5 and 6 as depicted in the following formulas in equation 7 .

$$
\left.(H \lambda) \times \frac{\partial k}{\partial t}(v, t)=(\bar{T} \lambda) \times\left[\int k(v 1, t) K(v)-k(v, t) K(v 1)\right) \sigma(v, v 1) d v 1\right]
$$

At this point, the total exit complexity should be realized as follow: Total Exit complexity (TEC) is the sum of both Egress Complexity and Kinetic Complexity. More expands of this equation appear by merging equation 4 and 7 . then (TEC) should be represented as follow:

$$
\begin{aligned}
& \left.T E C=(H \lambda) \times\left[\int k(v 1, t) K(v)-k(v, t) K(v 1)\right) \sigma(v, v 1) d v 1\right] \\
& -\sum_{k} P I \log _{2} \frac{P I}{r_{n}}-N I \log 2 \frac{N I}{r_{n}}
\end{aligned}
$$

By substituting the value of rd in equation 8 , then (TEC) is represented as follow:

$$
\begin{gathered}
\left.T E C=(H \lambda) \times\left[\int k(v 1, t) K(v)-k(v, t) K(v 1)\right) \sigma(v, v 1) d v 1\right]^{(9)} \\
-\sum P I \log 2 \frac{P I}{\sum_{k} \prod_{d=1}^{h-1}\left(\begin{array}{l}
\left.r_{d}+j_{d}-1\right) \\
t_{d}
\end{array}\right)} \\
\sum_{x} \prod_{d=1}^{h-1}\left(\begin{array}{l}
r d+j_{d}-1 \\
t_{d}
\end{array}\right)
\end{gathered}
$$

On other words, the total exit complexity is represented in the following equation: 


$$
\begin{aligned}
& T E C=\frac{O^{2}}{2 s a^{2}}\left[2 U \lambda_{n}-\sum_{a=1}^{U}\left(\lambda_{n-a}+\lambda_{n+a}\right)\right] \times\left[\begin{array}{l}
k(v 1, t) K(v) \\
-k(v, t) K(v 1)) \sigma(v, v 1) d v 1
\end{array}\right]
\end{aligned}
$$

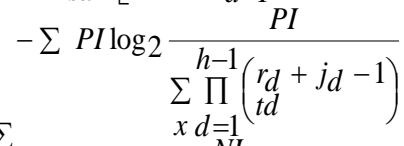

$$
\begin{aligned}
& \sum_{k N I \log _{2} \frac{x d=1}{N I}} \prod_{x d=1}^{h-1}\left(\begin{array}{r}
\left.r_{d}+j_{d}-1\right) \\
t_{d}
\end{array}\right)
\end{aligned}
$$

Equation 9 represents the total exit complexity in quantum distributed systems considering all the operators that can affect this complexity. The algorithm depicted in table 1 demonstrates the practical steps to calculate the complexity of knowledge in distributed quantum computer Systems. The algorithm depends on building bilateral non-tree entitled roots as a model of knowledge in distributed computing. In addition to some special accounts such as determining the length and the distance between each episode in the tree, and then switch between these episodes. We must put all storage nodes and the general structure of a binary tree. At this step has to be to search for positive and negative moments during the movement of knowledge. Then calculate the amount of information specific similarities in the system. In this case, the algorithm calculates the degree of complexity out information. It can then calculate the roots of the tree of others entitled, which was considered a step by calculating total egress complexity. The algorithm shows the penultimate step when calculating the degree of complexity. And forbids certain operations to compute the total exit complexity.

1- generate graph tree.

2- calculate the height of the tree.

3- moving between two adjacent nodes.

4- save the position of each node.

5- save the total schematic representation of the tree.

6- search for the total positive instances and the total negative instances.

7- measure the pseudo-information measure Is.

8- Calculate the information needed to egress from no exit node.

9- calculate the unlabeled rooted trees.

10 - measure the egress complexity.

11- measure the kinetic complexity.

12- Calculate the total exit complexity as

(kinetic complexity.+ egress complexity)

Table1 : the complexity algorithm of knowledge in distributed quantum computer systems

\section{B. Minimizing the exit complexity in distributed quantum computing systems}

In this section we discuss a methodology to minimize the exit complexity in distributed quantum computing systems. The main concept in minimizing the exit complexity is to portion the main system into subsystems logically. This portioning process contribute to reduce the different types of complexity in quantum systems. This concept of portioning is first introduced by Mingsheng Ying et al in [37]. Also they introduced basic algebraic laws for these groups of logical circuits using more detailed cases. This paper uses the algebraic formulas of division in subsystems and extend this work to study the effect of this division on the total exit complexity of the system. Mingsheng Ying et al in [37] consider CR as a circuit and $P=\left\{G_{t}: t \in E\right\}$ a partition of $\mathrm{D}(\mathrm{CR})$. They mention that $\mathrm{C}$ deference $\mathrm{P}$ when $\mathrm{C}$ deference that partition $P \cup\{G \backslash D(C)\}$. Where MQ1 and MQ2 be two sets of qubit names. If CR deference $P=\left\{G_{t}: t \in E\right\}$ and $G_{i} \subseteq M_{t i}$ (i=1,2). Then CR separates G1 from G2 both quantum measurements and unitary transformations can only be performed on local subsystems. Classical information extracted by a measurement on one subsystem can be passed to other subsystems. Also, entanglement resources are allowed to reside between different subsystems, and thus to connect them. In other words, many subsystems can share a single quantum resource in a distributed system. Now, consider TECn is the exit complexity for the $n$ circuit, and $\mathrm{Pn}$ is the fraction of the exit complexity related to the main system for the $\mathrm{n}$ circuit. To calculate the complexity at $\mathrm{n}$ circuit, the formula of TECn is represented as in equation 10:

$$
T E C_{n}=\frac{T E C}{P_{n}}(10)
$$

Each circuit in the subsystem will have few numbers of eignvalues $\lambda$. From equation 9 it is concluded that:

$$
\frac{\partial(\text { TEC })}{\partial(\lambda)}=\left[\frac{2 U O^{2}}{2 s a^{2} P_{n}}-\frac{\partial}{\partial \lambda} \sum_{a=1}^{U}\left(\lambda_{n-a}+\lambda_{n+a}\right)\right] \times\left[\begin{array}{l}
k(v 1, t) K(v) \\
-k(v, t) K(v 1)) \sigma(v, v 1) d v 1
\end{array}\right]=0
$$

$$
\begin{aligned}
& \text { Hence } \frac{2 U O^{2}}{2 s a^{2} P_{n}}=\frac{\partial}{\partial \lambda} \sum_{a=1}^{U}\left(\lambda_{n-a}+\lambda_{n+a}\right) \\
& P_{n=} \frac{2 U O^{2}}{2 s a^{2}\left(\frac{\partial}{\partial \lambda} \sum_{a=1}^{U}\left(\lambda_{n-a}+\lambda_{n+a}\right)\right)}(11)
\end{aligned}
$$

The formula of equation 11 presents the optimal value of Pn to obtain minimum values of the total exit complexity in logical subsystem. Table 2 depicts the algorithm used for An algorithm for minimizing the exit complexity in distributed quantum computing systems. It is depicted from this algorithm that is important steps to check various values of the circuits such as P , C and CR. This checking steps assure that the separation process is occurred.

1- Divide the main system logically into $\mathrm{CR} 1, \ldots . . \mathrm{CRn}$

2- Check for $\mathrm{C}$ deference $\mathrm{P}$ 
3- Check for $\mathrm{P}=\{\mathrm{Gt}: \mathrm{t}, \mathrm{E}\}$

4- Check that $\mathrm{C}$ deference $\mathrm{P}$ when $\mathrm{C}$ deference $\mathrm{P}$ union $\{\mathrm{G} \backslash \mathrm{D}(\mathrm{CR})$.

5- If ( $\mathrm{CR}$ deference $\mathrm{P}$ and $\mathrm{Gi}$ ) Then $\mathrm{CR}$ separates MQ1 from MQ2)

6- Calculate TECn as (TEC/Pn)

7- Set the derivation of TEC with respect to $\lambda$ to zero

8- Deduce the optimal value of Pn

1- Divide the main system logically into CR1,....CRn

2- Check for $\mathrm{C}$ deference $\mathrm{P}$

3- Check for $\mathrm{P}=\{\mathrm{Gt}: \mathrm{t}, \mathrm{E}\}$

4- Check that $\mathrm{C}$ deference $\mathrm{P}$ when $\mathrm{C}$ deference $\mathrm{P}$ union

$\{\mathrm{G} \backslash \mathrm{D}(\mathrm{CR})$.

5- If ( CR deference P and Gi) Then CR separates MQ1

from MQ2)

6- Calculate TECn as (TEC/Pn)

7- Set the derivation of TEC with respect to $\lambda$ to zero

8- Deduce the optimal value of Pn

Table2: An algorithm for minimizing the exit complexity in distributed quantum computing systems

\section{System Simulation AND Results}

Figure 2 shows that when calculating the egress complexity at low values for both positive and negative moments of the rate increases tree logarithm of these moments in a linear fashion, regular and value very small egress complexity and increasingly up to scratch positive moments. In the mean values for positive moments increase egress complexity values, especially when the supreme moments as shown in Figure 3. When the supreme values of positive and negative moments value of egress complexity increases in the beginning, but when you increase the number of positive and negative moments significantly up to a value of zero as shown in Figure 4. Deduct from figures 2, 3 and 4 that the egress complexity be less than what can be at the highest values for both positive and negative moments. Figures 2, 3 and 4 refer to number of guidelines such as PI represented as s1, NI represented as s2 and $\log 2(\mathrm{NI} / \mathrm{m})$ represented as $\mathrm{s} 3$. Information is also evident from the figures 5, 6 and 7 that the value of the kinetic operator increases with increasing values of both $\mathrm{h}, \mathrm{m}$, a, a transaction affecting the overall value for kinetic complexity therefore increases significantly increased values of kinetic operator. figures 5, 6 and 7 refer number of guidelines such as h represented as $\mathrm{s} 1, \mathrm{~m}$ represented $\mathrm{s} 2$, a represented as $\mathrm{s} 3, \mathrm{~N}$ represented as s4, n represented as s5, NI represented as s6, PI represented as $\mathrm{s} 7$ and $\mathrm{T}$ represented as $\mathrm{s} 8$. Evidenced by the figures 8,9 and 10 to exit complexity increases significantly when the supreme values of positive and negative moments of the tree that represents the volume of knowledge and significantly less at low values. Figures 8, 9 and 10 refer to the exit complexity as s1, egress complexity as s2 and kinetic operator as s3. For low values for exit complexity as shown in Figure 9 are working on the average values for higher values of positive and negative. From the above analysis this paper went to the importance of work to get to know on a regular basis with an average value for both the positive and negative moments to get the lowest value for the exit complexity. Practically These findings show the need to find a way to control the shape and structure of knowledge transmitted in distributed computing Quantity and control the speed of the transfer of this knowledge. Also end that we cannot separate the structure of knowledge and the complexity out of knowledge. The balanced structure of knowledge refers to the decline in values held out data from point to point in quantum systems.

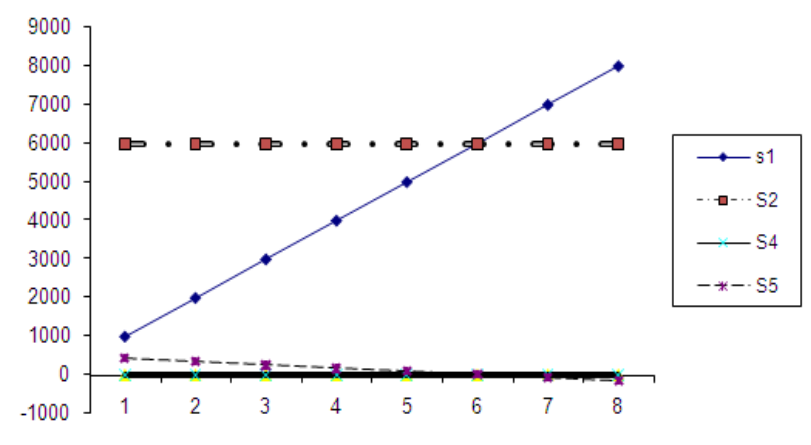

Fig.2 : Egress complexity at high values of PI and NI

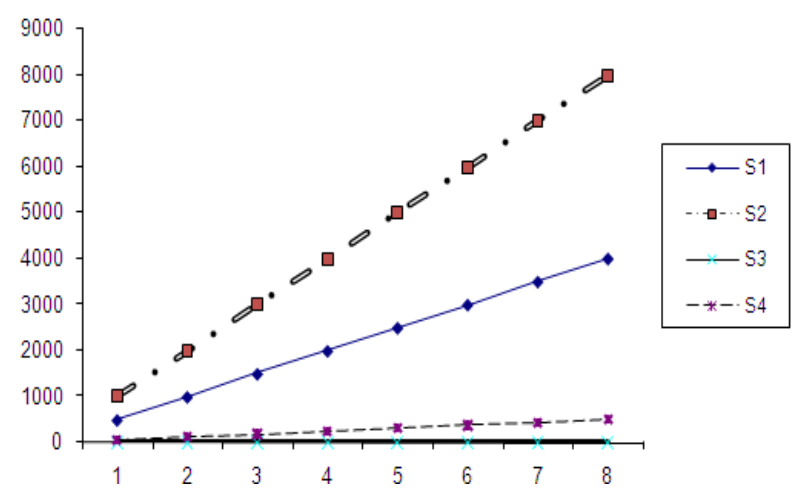

Fig. 3 : Egress complexity at intermediate values of PI and NI

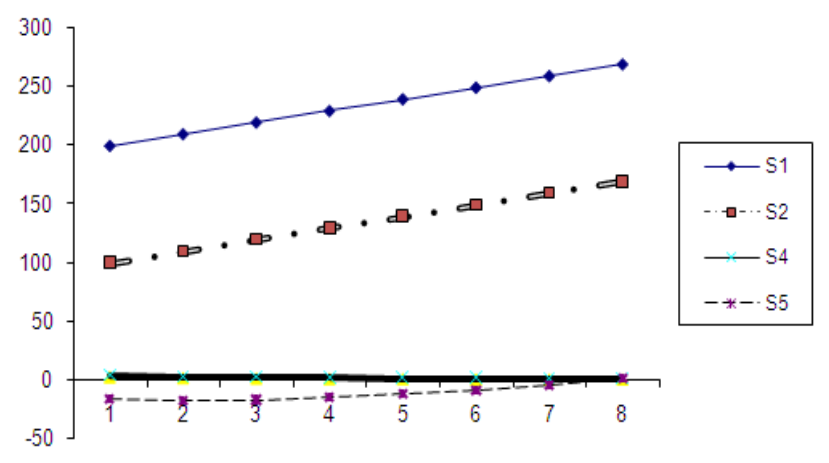

Fig. 4 : Egress complexity at low values of PI and NI 


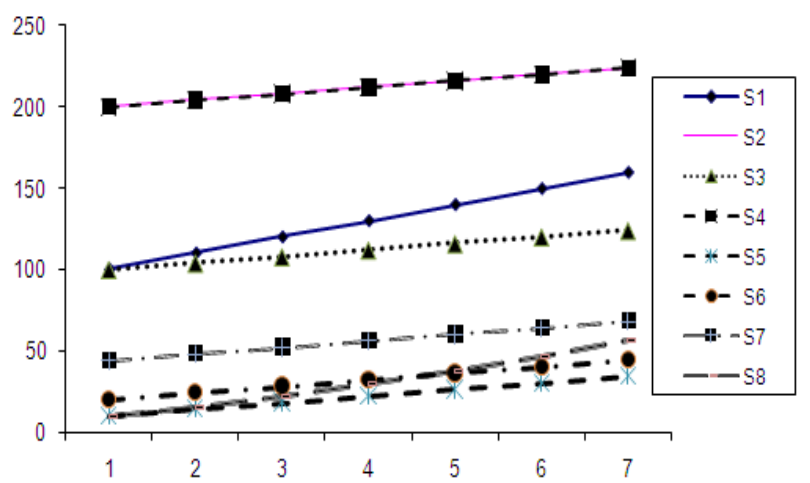

Fig.5 : kinetic operator at low values of NI and PI

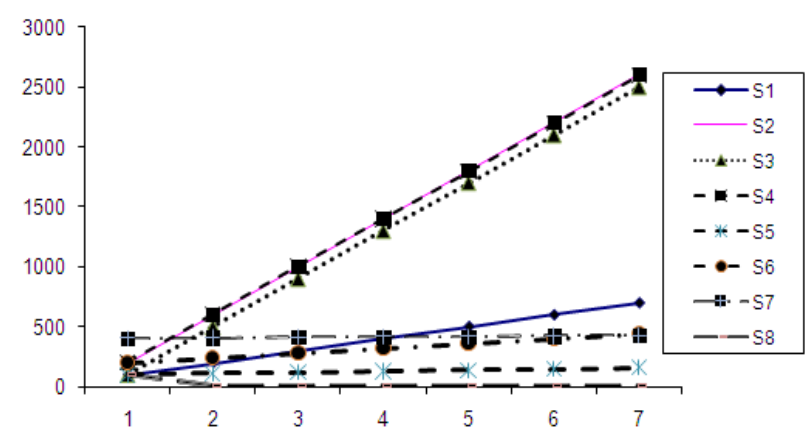

Fig. 6 : kinetic operator at intermediate values of NI and PI

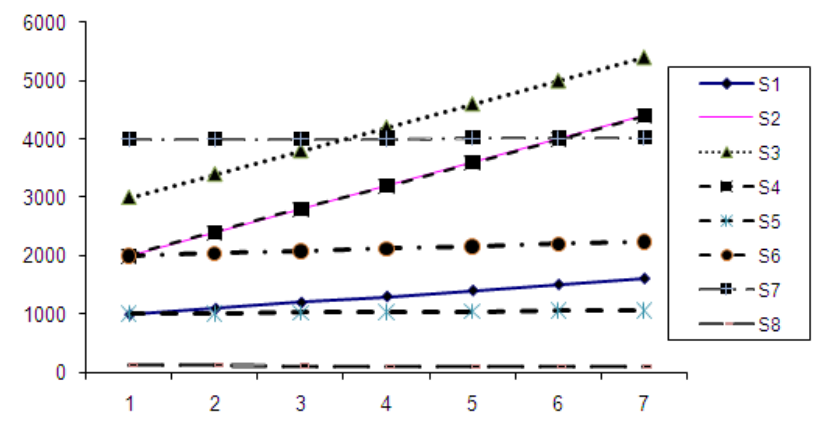

Fig.7 : kinetic operator at high values of NI and PI

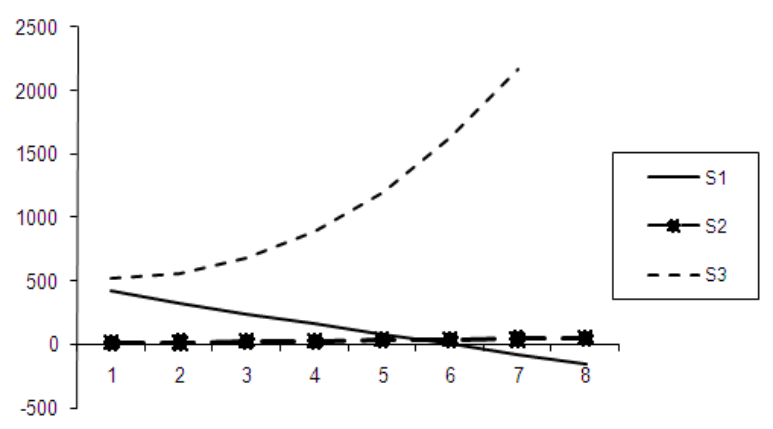

Fig.8 : Total Exit complexity at high values of egress complexity

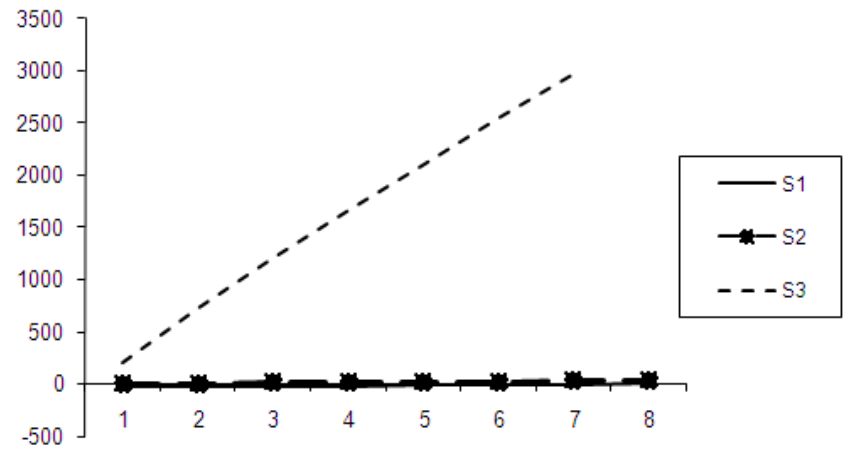

Fig.9 : Total Exit complexity at intermediate values of egress complexity

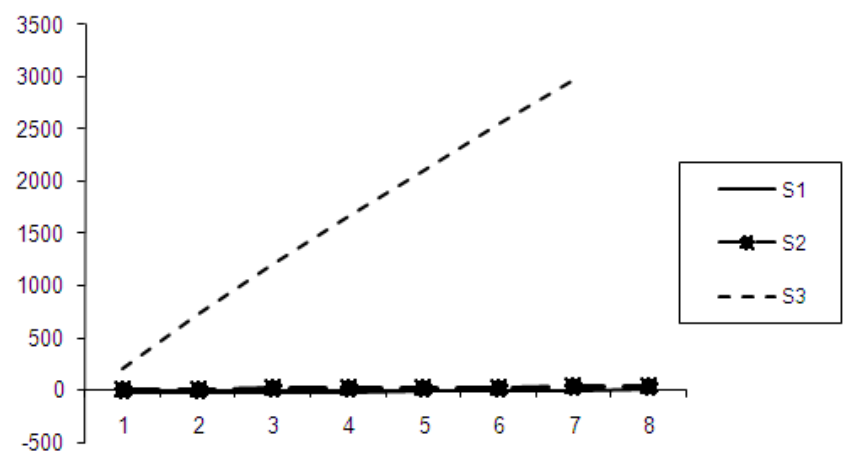

Fig.10 : Total Exit complexity at low values of egress complexity

\section{CONCLUSIONS}

A quantum computer is a computer design which uses the principles of quantum physics to increase the computational power beyond what is attainable by a traditional computer. Quantum computers have been built on the small scale and work continues to upgrade them to more practical models. Quantum computer's main drawback is the same as its strength: quantum coherence. The qubit calculations are performed while the quantum wave function is in a state of superposition between states, which is what allows it to perform the calculations using both one and zero states simultaneously. All the above drawbacks can lead to more complexity in the exit of knowledge in distributed quantum computers. This research sheds light on how to calculate the degree of complexity out knowledge in distributed computing quantum systems. Then both are calculated Unlabeled binary trees In this research methodology proposed to express their knowledge by building. Total exit complexity and then calculated Kinetic complexity and Egress complexity. This paper also suggested using a logical approach to minimize the total exit complexity for the main system. The results indicate that the egress complexity be less than what can be at the highest values for both positive and negative moments. The transaction affecting the overall value for kinetic complexity therefore increases significantly increased values of kinetic operator. The exit complexity increases significantly when the supreme values of positive and negative moments of the tree that represents the volume of knowledge and significantly less at low values.

\section{REFERENCES}

[1] http://en.wikipedia.org/wiki/Quantum_computer, visited 14-7-2012 
[2] Ellie D'Hondt, " Distributed quantum computation a measurementbased approach", Faculteit Wetenschappen , July 2005

[3] Samson Abramsky \& Bob Coecke. A categorical semantics of quantum protocols. In Proceedings of the 19th annual IEEE Symposium on Logic (LICS) in Computer Science. IEEE Computer Society, 2004.

[4] Pedro Ad ao \& Paulo Mateus. A process algebra for reasoning about quantum security. In Peter Selinger, Ed., Proceedings of the 3rd Workshop on Quantum Programming Languages (QPL04), pages 3-20, 2005.

[5] Panos Aliferis \& Debbie W. Leung. Computation by measurements: a unifying picture. Phys. Rev. A, 70:062314, 2004.

[6] Thorsten Altenkirch \& Jonathan Grattage. A functional quantum programming language. In Proceedings of the 20th Annual IEEE Symposium on Logic in Computer Science (LICS). IEEE Computer Society, 2005.

[7] Andris Ambainis, Harry Buhrman, Yevgeni Dodis, \& Hein R"ohrig. Multiparty quantum coin flipping. In Proceedings of the Conference on Computational Complexity (CCC), 2004.

[8] Dana Angluin. Local and global properties in networks of processors. In Proceedings of the 12th annual ACM symposium on Theory of computing, pages 82-93. ACM Press, 1980.

[9] Alexandru Baltag \& Sonja Smets. Quantum dynamic logic. In Peter Selinger, Ed., Proceedings of the 2nd Workshop on Quantum Programming Languages (QPL04), Turku, Finland, 2004. Turku Centre for Computer Science, TUCS General Publication No 33.

[10] Alexandru Baltag \& Sonja Smets. LQP: the dynamic logic of quantum information. Unpublished, 2005.

[11] Simon C. Benjamin, Daniel E. Browne, Joe Fitzsimons, \& John J.L. Morton. Brokered graph state quantum computing. 2005.

[12] Abrams D S and Lloyd S 1997 Simulation of many-body Fermi systems on a universal quantum computer Phys. Rev. Lett. 79 2586-9

[13] Aharonov D and Ben-Or M 1996 Fault-tolerant quantum computation with constant error Preprint quantph/ 9611025

[14] Aspect A, Dalibard J and Roger G 1982 Experimental test of Bell's inequalities using time-varying analysers Phys. Rev. Lett. 49 1804-7

[15] Barenco A 1995 A universal two-bit gate for quantum computation Proc. R. Soc. A 449 679-83

[16] Barenco A, Bennett C H, Cleve R, DiVincenzo D P, Margolus N, Shor P, Sleator T, Smolin J A and Weinfurter H 1995b Elementary gates for quantum computation Phys. Rev. A 52 3457-67

[17] Speakable and Unspeakable in Quantum Mechanics (Cambridge: Cambridge University Press) Benioff P 1980 J. Stat. Phys. 22563

[18] Bennett C H, Bernstein E, Brassard G and Vazirani U 1997 Strengths and weaknesses of quantum computing Preprint quant-ph/9701001

[19] Bennett C H, Brassard G, Briedbart S and Wiesner S 1982 Quantum cryptography, or unforgeable subway tokens Advances in Cryptology: Proceedings of Crypto '82 (New York: Plenum) pp 267-75

[20] Berman G P, Doolen G D, Holm D D, Tsifrinovich V I 1994 Quantum computer on a class of one-dimensional Ising systems Phys. Lett. 193 $444-50$
[21] Bernstein E and Vazirani U 1993 Quantum complexity theory Proc. 25th Annual ACM Symposium on Theory of Computing (New York: ACM) pp 11-20

[22] Berthiaume A and Brassard G 1992a The quantum challenge to structural complexity theory Proc. 7th Annual Structure in Complexity Theory Conf. (Los Alamitos, CA: IEEE Computer Society Press) pp 132-7

[23] Braunstein S L, Mann A and Revzen M 1992 Maximal violation of Bell inequalities for mixed states Phys. Rev. Lett. 68 3259-61

[24] Brune M, Nussenzveig P, Schmidt-Kaler F, Bernardot F, Maali A, Raimond J M and Haroche S 1994 From Lamb shift to light shifts: vacuum and subphoton cavity fields measured by atomic phase sensitive detection Phys.

[25] qubit-external.physics.ox.ac.uk/...quantum-computing/../20-quantumcomputing-longer-intro.htm, visited 24-8-2012

[26] Simon D, "On the power of quantum computation", in Proc. 35th Annual Symposium on Foundations of Computer Science (IEEE Computer Society Press, Los Alamitos), 1994 , 124-134

[27] Shor P W ," Polynomial-time algorithms for prime factorization and discrete logarithms on a quantum computer", in Proc. 35th Annual Symp. on Foundations of Computer Science, Santa Fe, IEEE Computer Society Press; revised version 1995a preprint quantph/ 9508027, 1994

[28] Kitaev A Yu," Quantum measurements and the Abelian stablizer problem", (preprint quantph/ 9511026), 1995

[29] Beckman D, Chari A, Devabhaktuni S and Preskill J " Efficient networks for quantum factoring", Phys. Rev. A 54, 1034-1063, 1996

[30] Alexandru Baltag \& Sonja Smets. "Quantum dynamic logic". In Peter Selinger, Ed., Proceedings of the 2nd Workshop on Quantum Programming Languages (QPL04), Turku, Finland, 2004. Turku Centre for Computer Science, TUCS General Publication No 33.

[31] Vincent Danos, Ellie D'Hondt, Elham Kashefi, \& Prakash Panangaden." Distributed measurement-based quantum computation". In Peter Selinger, Ed., Proceedings of the 3rd Workshop on Quantum Programming Languages (QPL05), 2005. quant-ph/0506070.

[32] Ron van der Meyden \& Manas Patra. Knowledge in quantum systems. In Proceedings of the 9th conference on Theoretical aspects of rationality and knowledge, pages 104-117, Bloomington, Indiana, June 2003.

[33] Hongjun Jiang, "The development of a scenario Independent Method for Evaluating the Evacuation Complexity of a building", March 2012, The university of Greenwish

[34] H. A. DONEGAN, "Formal Aspects of Egress Complexity", Mathematical and Computer Modelling 35 (2002) 119-128

[35] Gang Li , " Generation of Rooted Trees and Free Trees", University of Victoria 1996.

[36] Christian Lécot et al, " Quasi-random Simulation of Linear Kinetic Equations", journal of complexity 17, 795-814 (2001)

[37] Mingsheng Ying and Yuan Feng, "An Algebraic Language for Distributed Quantum Computing" IEEE Transactions on Computers, VOL. 58, NO. 6, JUNE 2009 